\title{
Der rechtliche Rahmen der Verwaltungskommunikation
}

\author{
Till Dunckel
}

\section{$1 \quad$ Einführung}

Die Ausübung von Staatsgewalt war schon immer mit der Aufgabe verbunden, sie gegenüber der Öffentlichkeit zu kommunizieren. Dies gilt keineswegs nur für die Regierungsspitzen, sondern auch für die Administration: Möchte eine Behörde beispielsweise Schwimmbäder schließen, Verkehr beruhigen oder Öffnungszeiten ändern, ist sie auf eine funktionierende Kommunikation gegenüber und mit den Bürgern angewiesen.

Die systematische Öffentlichkeitsarbeit der Exekutive ist also kein Phänomen der heutigen Medienwelt. Diese hat jedoch die Quantität und Intensität der Verwaltungskommunikation erhöht und schließlich dazu beigetragen, dass diese in den vergangenen Jahren Gegenstand einer zunehmenden rechtlichen Regelungsdichte - sowohl durch gesetzliche Vorgaben als auch durch gerichtliche Entscheidungen - geworden ist.

Im Anschluss an die Darstellung einiger grundlegender Aspekte stellen wir im Folgenden die wichtigsten juristischen Aspekte der behördlichen Öffentlichkeitsarbeit zusammen. Hier werden zunächst die wichtigsten Regelungen erörtert, nach denen die Verwaltung zur (öffentlichen) Kommunikation verpflichten sein kann. Anschließend wird dargelegt, unter welchen Voraussetzungen eine Behörde berechtigt ist, sich auch ohne Verpflichtung aktiv an die Öffentlichkeit zu wenden. Und schließlich erläutern wir, welche rechtlichen Grenzen für die Inhalte der Verwaltungskommunikation gelten.

T. Dunckel $(\bowtie)$

Nesselhauf Rechtsanwälte, Hamburg, Deutschland

E-Mail: dunckel@nesselhauf.com 


\section{$2 \quad$ Allgemeine Fragen}

Kommunikation in der Praxis Während die Begriffe ,Kommunikation“ und ,Öffentlichkeitsarbeit' als konturenlose Sammelbegriffe sämtliche Arten (öffentlicher) Mitteilungen umfassen, haben sich in der Praxis unterschiedliche Praktiken herausgebildet, über die die Exekutive Informationen an die Öffentlichkeit verbreiten kann.

Beantwortung von (Presse-) Anfragen Die Beantwortung von Presseanfragen ist in der Praxis die häufigste und zugleich die passivste Variante öffentlicher Kommunikation. Denn die Behörde kann im Normalfall weder entscheiden, wann und welche Fragen ihr gestellt werden, noch kann sie aufgrund zahlreicher gesetzlich normierter Auskunftspflichten frei entscheiden, ob und wie sie die Fragen beantwortet.

Pressemitteilungen Anders als bei der Beantwortung individueller Anfragen geht bei der Verbreitung von Pressemitteilungen die Initiative von der Behörde aus. Sie entscheidet im Rahmen ihres gesetzlichen Spielraums, ob, zu welchem Zeitpunkt und mit welchem Inhalt sie sich zu einem Sachverhalt öffentlich äußert.

Pressekonferenzen und Pressegespräche Wenn und soweit Informationen per Pressemitteilung verbreitet werden dürfen, kann dies grundsätzlich auch durch öffentliche Pressekonferenzen oder sogenannte Pressegespräche, einem Austausch mit einer kleineren Gruppe ausgewählter Journalisten, geschehen.

Interviews Presseinterviews, bei denen sich ein Vertreter einer Behörde den Fragen (in der Regel) eines Journalisten stellt, bieten der Behörde aufgrund ihres Dialogformats einerseits die Chance, die behandelten Sachverhalte substanziiert zu erläutern. Auf der anderen Seite steht das erhebliche Risiko, dass das Gespräch durch überraschende oder kritische Fragen einen anderen als den von der Behörde beabsichtigten Verlauf nimmt. Der in der Praxis häufige Versuch, dieses Risiko durch Interviewvereinbarungen zu umgehen, die dem Interviewten das Recht geben, ganze Frage/Antwort-Komplexe zu streichen, widerspricht dem Selbstverständnis der meisten Redaktionen und ist insbesondere im Bereich der Kommunikation durch Hoheitsträger allenfalls mit Zurückhaltung und Fingerspitzengefühl zu nutzen.

Hintergrundgespräche Hintergrundgespräche dienen dazu, Informationen an Medienvertreter weiterzugeben, ohne hierbei öffentlich als Quelle in Erscheinung 
zu treten. Faktisch bietet dies der Behörde den Vorteil, ihre Sicht der Dinge zu erläutern und auf Nachfragen reagieren zu können, ohne jedes Wort in der gleichen Weise juristisch und politisch abwägen zu müssen, wie dies in den zuvor genannten Kommunikationsformen der Fall ist.

Gesetzlich geregelt sind Hintergrundgespräche nicht. Da sie letztlich dazu dienen, auf verdeckte Weise, aber gleichwohl unter Inanspruchnahme behördlicher Autorität, Informationen zu platzieren, ist ihre Vereinbarkeit mit dem Rechtsstaatsgebot jedenfalls nicht selbstverständlich.

\section{Nicht-amtliche Äußerungen von Amtsträgern}

Die besonderen juristischen Voraussetzungen öffentlicher Verwaltungskommunikation gelten nur, wenn die Öffentlichkeitsarbeit dem Staat zuzurechnen ist. Dies ist in der Praxis meist unproblematisch, wenn die öffentliche Äußerung ausdrücklich (beispielsweise bei schriftlichen Erklärungen durch den Briefkopf einer Behörde) erfolgt oder die äußeren Umstände keinen Zweifel lassen, dass die Öffentlichkeitsarbeit - wie beispielsweise bei einer Äußerung des Polizeipressesprechers vor laufenden Kameras - im Namen der handelnden Behörde erfolgt (Debus 2017; IFG § 1 Rn. 118 ff.).

Allerdings stellen sich in der Praxis immer wieder Grenzfälle zur Entscheidung, in denen die richtige Zuordnung einer öffentlichen Äußerung schwerfällt. Dies ist insbesondere dann der Fall, wenn ein öffentlicher Amtsinhaber zugleich in einer privatrechtlichen Organisation eine exponierte Position bekleidet. Bei Spitzenpolitikern, die neben ihren öffentlichen Ämtern auch Parteiämter ausüben, ist dies regelmäßig der Fall (Barczak 2015, S. 1015).

In diesen Grenzfällen kommt es für die richtige Zuordnung zum einen darauf an, ob der sich Äußernde innerhalb des ihm zugewiesenen Aufgaben- und Zuständigkeitsbereich gehandelt hat. Zum anderen hängt die Zuordnung davon $\mathrm{ab}$, ob er aus Sicht der Empfänger als Behördenvertreter gehandelt hat. Hier hängt die Entscheidung auch von äußeren Umständen, wie beispielsweise dem organisatorischen Rahmen, die den Adressaten mitgeteilte Tätigkeit oder die Räumlichkeiten einer Presseveranstaltung, ab (Barczak 2015, S. 1016). Wenn die Kommunikation aus Sicht der Empfänger die mit einem öffentlichen Amt verbundene Sachlichkeits- und Richtigkeitserwartung für sich in Anspruch nimmt, kann an deren Zuordnungen als behördliche Äußerung kein Zweifel bestehen (Barczak 2015, S. 1016). 


\section{$4 \quad$ Gleichbehandlung im publizistischen Wettbewerb}

Aus dem Zusammenspiel der Medienfreiheiten des Artikel 5 I GG mit dem allgemeinen Gleichheitssatz aus Artikel 3 I GG folgt ein Anspruch der Medien auf Gleichbehandlung im publizistischen Wettbewerb. Diese Neutralitätspflicht verpflichtet eine Behörde, Medienvertreter bei der Verbreitung von Informationen ohne besondere, sachliche Gründe nicht unterschiedlich zu behandeln.

Bei der freiwilligen Verbreitung von allgemeinen Pressemitteilungen muss eine Behörde diese daher sämtlichen interessierten Medienvertretern ohne Rücksicht auf sachliche oder persönliche Qualifikationen in gleicher Weise zugänglich machen; sämtliche Presseorgane sind hinsichtlich des Zeitpunkts, Umfangs und Inhalts der ihnen zugänglich gemachten Informationen strikt gleich zu behandeln (BVerfG ZUM 1990, 130; BVerwG ZUM 1998, 78; OVG NW, ZUM-RD 2000, 253); dies gilt über das amtliche Bekanntmachungen betreffende (beispielsweise in § 4 IV LPG und § 9a III RStV) normierte Benachteiligungsverbot hinaus grundsätzlich für jede Art der Öffentlichkeitsarbeit. Dementsprechend müssen sämtliche interessierte Medienvertreter die Möglichkeit erhalten, an einer behördlichen Pressekonferenz teilzunehmen.

Schwieriger gestaltet sich die Gleichbehandlung im publizistischen Wettbewerb bei der Durchführung von Pressegesprächen: Einerseits erkennt die Rechtsordnung an, dass der Grundsatz strikter Gleichbehandlung nicht dazu führen darf, dass nur noch eine ,uniforme Massenunterrichtung der Presse in Form allgemeiner Pressekonferenzen“ (VG Bremen, NJW 1997, 2696, 2698) möglich wäre. Auch im Lichte des Gleichbehandlungsgrundsatzes soll es der Verwaltung möglich sein, Sachverhalte in einem persönlich überschaubaren Kreis von Journalisten dazustellen und zu erläutern (BVerwG, NJW 1975, 891, 892; Löffler et al. 2015, PresseR, § 4 LPG Rn. 143). Andererseits muss die Auswahl der Teilnehmer eines Gesprächskreises anhand ausnahmslos sachgerechter Kriterien erfolgen. Keine geeigneten Sachkriterien wären beispielsweise das persönliche Vertrauensverhältnis zu einzelnen Journalisten oder die subjektive Einschätzung der publizistischen Relevanz eines Mediums.

Bei der Durchführung eines (Einzel-)Interviews ist die Ungleichbehandlung der Medien sogar wesensimmanent. Gleichwohl gilt auch hier, dass diese Ungleichbehandlung im Interesse einer substanziierten Information der Öffentlichkeit möglich sein muss. Allerdings bedarf es auch bei der Durchführung von Einzelinterviews sachgerechter Kriterien, die die Auswahl des Mediums objektiv nachvollziehbar machen. Gegen den Verdacht behördlicher Willkür spricht zudem ein Rotationssystem, das andere sachlich geeignete Redaktionen bei zukünftigen Interviews oder Pressegesprächen berïcksichtigt. 


\section{Maßgebliches Verständnis einer Äußerung}

Vor der rechtlichen Qualifikation einer Äußerung ist es zunächst erforderlich, ihren Aussagegehalt richtig zu erfassen. Zugrunde zu legen ist dabei stets das Verständnis, das ein unvoreingenommenes und verständiges Publikum aus der fraglichen Äußerung erlangen würde. Dabei ist stets zu berücksichtigen, in welchem Gesamtzusammenhang die Äußerung gefallen ist (BGH ZUM 2018, 527 Rn. 20). Eine vom Kontext gelöste, isolierte Betrachtung des Inhalts einer Äußerung ist unzulässig. Unerheblich ist bei der Ermittlung des Aussagegehalts, was der Äußernde mitteilen wollte. Darüber hinaus ist das Verständnis nicht auf den Wortlaut der Äußerung beschränkt: Insbesondere sind auch Äußerungen ,zwischen den Zeilen' (so genannte verdeckte Äußerungen) der juristischen Prüfung zugrunde zu legen, wenn ein hinreichender Teil der Leser einer Äußerung diese verdeckte Mitteilung entnimmt.

\section{$6 \quad$ Internet und soziale Medien}

Für die rechtliche Betrachtung von Kommunikationspflichten und Kommunikationsrechten der Behörden ist die technische Verbreitungsform grundsätzlich unerheblich. Für die Kommunikation in einer Pressekonferenz gelten grundsätzlich keine anderen Regelungen als für eine Bürgerinformation über WhatsApp, Twitter oder andere moderne Kommunikationskanäle. Bei der Ermittlung des Aussagegehalts einer Äußerung oder der von ihrer faktischen Wirkung abhängigen Abwägung mit den Rechten Betroffener kann es hingegen durchaus auf die Besonderheiten des gewählten Kommunikationswegs ankommen.

\section{$7 \quad$ Auskunftspflichten der Verwaltung}

Auf der einen Seite der juristischen Betrachtung von Verwaltungskommunikation steht die Frage, ob und gegebenenfalls unter welchen Voraussetzungen eine Behörde verpflichtet sein kann, Kommunikation zu betreiben.

Dabei steht schon aus verfassungsrechtlichen Gründen außer Frage, dass Behörden grundsätzlich verpflichtet sind, die Öffentlichkeit über wichtige Sachverhalte zu informieren. Schließlich ist die Möglichkeit, staatliches Handeln effektiv kontrollieren zu können, ein wichtiges Merkmal des Rechtsstaats. Zum Zwecke der demokratischen Meinungs- und Willensbildung ist das Auskunftsrecht gegenüber 
Behörden daher von großer Bedeutung. Dabei dürfen aber nicht die Grundsätze des Aktengeheimnisses und der Vertraulichkeit der Verwaltung außer Acht gelassen werden. Für die im Grundgesetz hervorgehobene Aufgabe der Presse, aufzudecken, $\mathrm{zu}$ informieren und $\mathrm{zu}$ kritisieren, sind spezielle Informationsrechte erforderlich (Schnabel 2016, HmbPrg, § 4 Rn. 1; BVerfGE 20, 162, 176).

Demgegenüber stehen aber zum einen die Rechte privater Personen und Unternehmen: Wenn die begehrte behördliche Auskunft sie betrifft, steht die grundsätzliche Auskunftspflicht im unmittelbaren Konflikt mit dem allgemeinen Persönlichkeitsrecht aus Art. 12 I, aus 14 GG und aus anderen verfassungsrechtlichen Abwehrrechten von natürlichen Personen und (über Art. 19 IV GG) privaten Unternehmen (bspw. in $\S 4$ II Nr. $3 \mathrm{HmbPrG}$ ). Zum anderen können die Auskunftspflichten ihre Grenzen auch in der Funktionsfähigkeit der Behörde finden: Dies ist insbesondere der Fall, wenn die Offenbarung behördeninterner Vorgänge (wie beispielsweise die Bekanntgabe geheimer Ermittlungen) die effektive Aufgabenerfüllung der der Behörde gesetzlich zugewiesenen Aufgaben behindern würde (bspw. in § 4 II Nr. $2 \mathrm{HmbPrG}$ ).

Jede öffentliche Kommunikation durch eine Behörde setzt somit einen sorgfältigen Abgleich der zu veröffentlichenden Inhalte mit etwaigen berechtigten Privatinteressen sowie eigenen berechtigten Interessen der Behörde voraus. Zur Lösung dieses Anwendungskonflikts hat der Gesetzgeber in der jüngeren Vergangenheit zahlreiche gesetzliche Regelungen geschaffen.

\section{8 § 4 der Landespressegesetze}

Die Pflicht der Behörden zur Auskunftserteilung ist in verschiedenen gesetzlichen Bestimmungen geregelt. Die älteste dieser Regelungen findet sich in den $\S 4$ der Landespressegesetze. Wenngleich sich die Formulierungen in einigen Ländergesetzen unterscheiden, ist der Regelungsgehalt der Vorschriften im Wesentlichen vergleichbar.

Anspruchsberechtigte Auf den in $\S 4$ der Landespressegesetze normierten, sogenannten journalistischen Auskunftsanspruch können sich Vertreter der Presse (und teilweise auch des Rundfunks) berufen. Dabei ist der Umfang der Berechtigten weit zu verstehen. So soll der Anspruch jedem zustehen, der an der Erfüllung der öffentlichen Aufgabe der Presse mitwirkt (Schnabel 2016; HmbPrg, $\S 4$ Rn. 3; Paschke et al. 2016; § 4 HbgPresseG Rn. 2). Hierzu gehören Redakteure, Herausgeber, Verleger und freie journalistische Mitarbeiter. Zum Nachweis der Pressezugehörigkeit genügt die Vorlage des Presseausweises oder eines 
Schreibens der Redaktion (LG München I BeckRS 2008, 2775). Eine Begründung des Anspruchs ist nicht erforderlich, die begehrte Auskunft muss lediglich der Erfüllung der öffentlichen Aufgabe dienen (Schnabel 2016; § 4 Rn. 11).

Zur Presse zählen neben den periodisch erscheinenden Druckwerken grundsätzlich auch andere Medienorgane, die der in $\$ 3$ der Landespressegesetze normierten Aufgabe der Beschaffung und Verbreitung von Nachrichten, der Stellungnahme, der Kritik und der sonstigen Mitwirkung an der Meinungsbildung dienen. Die mitunter geführte Diskussion, ob auch Online-Medien oder bestimmte Formen des Rundfunks ,Presse“ im Sinne der Landespressegesetze (in § 4 HmbgPresseG ist der „Rundfunk“ sogar ausdrücklich genannt) sind, ist praktisch bedeutungslos, da sich in $\S 9$ a des Rundfunkstaatsvertrags (RStV) für den Rundfunk eine entsprechende Regelung befindet, die gemäß $§ 55$ Abs. 3 RStV auch für Telemedien gilt. Aufgrund des publizistischen Neutralitätsgebots ist es darüber hinaus nicht von Bedeutung, ob es sich bei ,der Presse' um vermeintlich hochwertige und seriöse Medien handelt (VG Hamburg AfP 2009, 296 ff. Rn. 37). Im Hinblick auf die durch das Internet bedingte weltweite Verfügbarkeit und der Internationalisierung der Berichterstattung wird auch eine Begrenzung auf die inländische Presse überwiegend abgelehnt (Schnabel 2016; § 4 Rn. 5).

Anspruchsverpflichtete Der in den Landesgesetzen normierte Informationsanspruch wurde jahrzehntelang wie selbstverständlich auch gegenüber den in dem jeweiligen Bundesland ansässigen Bundesbehörden angewendet. Diese Praxis hat das Bundesverwaltungsgericht im Jahre 2013 für rechtswidrig erklärt: Eine auf die Landespressegesetze gestützte Auskunftspflicht der Bundesbehörden ist seitdem ausgeschlossen. Im Übrigen ist in Bezug auf den Begriff der ,Behörde ' aber von einem weiten, medienrechtlichen geprägten Verständnis auszugehen: Denn wenn öffentliche Mittel eingesetzt werden, um staatliche Aufgaben zu erfüllen, kommt unabhängig von der formalrechtlichen Einkleidung ein berechtigtes öffentliches Informationsinteresse in Betracht (Paschke et al. 2016; §4 HmbPresseG Rn. 4). Beliehene und Stellen der mittelbaren Staatsverwaltung (Körperschaften, Anstalten, Stiftungen des öffentlichen Rechts) sind unproblematisch als Behörden erfasst (Löffler et al. 2015, PresseR, § 4 LPG Rn. 62).

Auskunftspflichtig sind grundsätzlich auch Organe der Legislative und der Judikative. Kirchen sind zur Auskunft verpflichtet, wenn die Fragen staatliche Angelegenheiten (also beispielsweise die von der Finanzverwaltung eingezogene Kirchensteuer) betreffen. Auch öffentlich-rechtliche Rundfunkanstalten können ausnahmsweise auskunftspflichtig sein, wenn sie wie beim Einzug von Rundfunkgebühren wie eine Behörde tätig werden (BVerfG-K NJW 1988, 382; BVerwGE 70, 310 ff.). Eine Besonderheit besteht für juristische Personen des 
Privatrechts, die von öffentlicher Hand beherrscht und zur Erfüllung öffentlicher Ausgaben eingesetzt werden (BGH NJW 2005, 1720 f.). Eine Beherrschung liegt regelmäßig dann vor, wenn mehr als die Hälfte der Anteile im Eigentum der öffentlichen Hand stehen (VG Berlin ZUM-RD 2013, 38, 41). Ist dies der Fall, so kann ein journalistischer Auskunftsanspruch auch gegen eine juristische Person des Privatrechts gerichtet werden.

Anspruchsinhalt Inhalt des Anspruchs ist vor allem das Recht auf eine sachgerechte, wahrheitsgemäße und vollständige Auskunft (OVG Bremen NJW 1989, 926; VGH München NJW 2004, 3358, 3359). Unerheblich ist dabei, welche Inhalte die Presse zur Erfüllung ihrer öffentlichen Aufgabe benötigt und welche Sachverhalte die Presse für berichtenswert hält; diese Entscheidung ist Teil des Kerns ihrer von Artikel 5 I GG geschützten Pressefreiheit und keiner Kontrolle durch die Verwaltung oder die Gerichte zugänglich. Hingegen erstreckt sich der Auskunftsanspruch regelmäßig nicht auf eine Aushändigung von Unterlagen (BVerwG NJW 2014, 1126 ff.; VG Mainz 14.09.2016 - 3 K 1021/15. MZ Rn. 26 ff.). Bei der Entscheidung, auf welche Art die Behörde die Auskunft erteilt, steht ihr ein weites Ermessen zu. Es steht ihr frei, eine Anfrage durch ein mündliches oder schriftliches Interview zu beantworten, zu einer Pressekonferenz einzuladen oder eine Pressemitteilung zu verbreiten.

Anspruchsgrenzen Das Gesetz nennt in Absatz 2 verschiedene Grenzen des Auskunftsanspruchs. Ob diese vorliegen, bedarf der Abwägung im Einzelfall, bei deren Ausübung der Behörde Ermessen zusteht (Paschke et al. 2016, § 4 HmbPresseG Rn. 11).

Demnach kann eine Behörde der Presse Auskünfte verweigern, wenn deren Erteilung die sachgemäße Durchführung eines schwebenden Gerichtsverfahrens, Bußgeldverfahrens oder Disziplinarverfahrens beeinträchtigen oder gefährden könnte. Erforderlich ist, dass die Verfahren noch nicht abgeschlossen sind und die Gefahr bereits konkret vorliegt (Schnabel 2016, § 4 Rn. 13). Außerdem können Auskünfte verweigert werden, wenn Vorschriften über die Geheimhaltung oder die Amtsverschwiegenheit entgegenstehen. Dabei muss es sich um Vorschriften handeln, die wie beispielsweise §353d StGB oder § 43 DRiG öffentliche Geheimnisse schützen und zumindest auch die Behörde zum Adressaten haben (OVG Hamburg AfP 2010, 617 Rn. 33). Schließlich kann die Auskunft verweigert werden, wenn die Erteilung der Auskunft ein „überwiegendes“ öffentliches (beispielsweise die innere Sicherheit, die Landesverteidigung, internationale Beziehungen) oder schutzwürdiges privates Interesse (beispielsweise der Schutz des informationellen Selbstbestimmungsrechts nach Art. 2 Abs. 1 iVm 
Art. 1 Abs. 1 GG oder der Schutz von Betriebs- und Geschäftsgeheimnissen) verletzen würde. Voraussetzung einer Auskunftsverweigerung ist jedoch stets das im Einzelfall festzustellende „Überwiegen“ dieses Rechts sowie die Prüfung, ob diesem entgegenstehenden Recht nicht auf andere Weise als die Auskunftsverweigerung (beispielsweise durch eine anonymisierte Auskunft) hinreichend Rechnung getragen werden kann.

\section{Verfassungsunmittelbarer Auskunftsanspruch gegenüber Bundesbehörden}

Seitdem das Bundesverwaltungsgericht im Jahre 2013 eine Anwendung der landespressegesetzlichen Auskunftsansprüche auf Bundesbehörden ausgeschlossen hat, leitet die Rechtsprechung eine eigene Auskunftspflicht der Bundesbehörden unmittelbar aus der Verfassung ab (BVerwGE 146, 56 ff.). Deren Umfang wurde in den letzten Jahren kontinuierlich ausgeweitet und unterscheidet sich mittlerweile kaum noch vom Umfang der landesgesetzlich garantierten Ansprüche (Schnabel 2016, S. 1692 ff.).

\section{$10 \S 9 \mathrm{a}$ Rundfunkstaatsvertrag}

§ 9a RStV regelt zugunsten von Rundfunkanbietern einen eigenen Auskunftsanspruch, der in Umfang und Grenzen mit den landespressegesetzlichen Ansprüchen vergleichbar ist. Gemäß $§ 55$ Abs. 3 RStV gilt dieser Auskunftsanspruch auch zugunsten von Telemedien.

\section{Die Informationsfreiheitsgesetze}

Die Rechtslage und das Selbstverständnis deutscher Behörden war über Jahrzehnte von dem Grundsatz geprägt, dass ein unbeteiligter Bürger nur dann Anspruch auf Auskunft zu behördeninternen Informationen hat, wenn er ausnahmsweise ein berechtigtes Interesse nachweisen kann. Mit der Verabschiedung mehrerer gesetzlicher Informationsfreiheitsgesetze wurde dieser Grundsatz in den vergangenen Jahren förmlich auf den Kopf gestellt. Nach der inzwischen geltenden Rechtslage sind Behörden grundsätzlich gegenüber jedem Bürger zur Auskunft verpflichtet, wenn der Informationserteilung nicht im Einzelfall gewichtige Gründe entgegenstehen. 
IFG und Landes-IFGe Auf Bundesebene gewähren das IFG und auf Länderebene die entsprechenden Landesregelungen (wie beispielsweise das HmbIFG) jedem Bürger und jeder juristischen Person einen nahezu voraussetzungslosen Anspruch auf Information über alle amtlichen Informationen, die sich im Besitz einer Behörde oder staatlicher Organe oder Einrichtungen der öffentlich-rechtlichen Verwaltung (Debus 2017, IFG, § 1 Rn. 118) befinden.

Dem sehr weitgehenden Anwendungsbereich dieser Informationsrechte stehen jedoch (beispielsweise in den §§ 3-6 IFG) zahlreiche Ausschlussgründe entgegen. Diese enthaltenen detaillierte Bestimmungen darüber, wann etwa zum Schutz von besonderen öffentlichen Belangen, zum Schutz des behördlichen Entscheidungsprozesses, zum Schutz personenbezogener Daten Dritter, zum Schutz des geistigen Eigentums Dritter oder zum Schutz privater Betriebs- und Geschäftsgeheimnissen Informationsgesuche zurückgewiesen werden können. Soweit die begehrte Information die Interessen Dritter berührt, ist die Behörde zudem verpflichtet, diesem vor einer Entscheidung über die Gewährung des Informationszugangs Gelegenheit zur Stellungnahme zu geben.

Das Umweltinformationsgesetz (UIG) Ziel des UIG ist die „Herstellung von Transparenz im Bereich der mit dem Thema Umwelt befassten Behörden und in staatlichen Auftrag tätigen Stellen“ (Karg 2018; UIG, Abschnitt 1, § 1). Hierfür sieht das UIG die passive Zugangsgewährung ( 33 UIG) und aktive Verbreitung (§ 10 UIG) von Umweltinformationen gegenüber der Öffentlichkeit vor. Der Anspruch auf Zugangsgewährung gilt voraussetzungslos für , jedermann'. Inhaltlich gehören dazu Daten über den Zustand der Umwelt, Faktoren, die sich auf den Zustand auswirken, Maßnahmen und Tätigkeiten, die sich auf die Umwelt auswirken oder ihren Schutz bezwecken, und Auswirkungen von Umweltveränderungen auf die menschliche Gesundheit und Sicherheit, sowie den Zustand von Kulturstätten und Bauwerken. Gem. § 3 UIG kann der Zugang durch Auskunftserteilung, Gewährung von Akteneinsicht oder in sonstiger Weise beantragt werden und nur aus gewichtigem Grund auf andere Art eröffnet werden. Die Grenzen des Anspruchs ähneln denen des IFG.

Das Verbraucherinformationsgesetz (VIG) Das VIG gewährt Verbrauchern freien Zugang gegenüber Bundes- und Landesbehörden und - soweit durch Landesrecht übertragen - auch gegenüber Gemeinden und Gemeindeverbänden zu Informationen über Erzeugnisse im Sinne des Lebensmittel- und Futtermittelgesetzbuches sowie über Verbraucherprodukte, die dem $\S 2$ Nr. 26 des Produktsicherheitsgesetzes unterfallen. Ziel des Gesetzes ist es gem. § $1 \mathrm{Nr}$. 2 VIG den „Markt transparenter zu gestalten und hierdurch den Schutz der Verbraucher vor 
gesundheitsschädlichen oder sonst unsicheren Erzeugnissen und Verbraucherprodukten sowie vor Täuschung beim Verkehr mit Erzeugnissen und Verbraucherprodukten“ zu verbessern. Die Ausschlussgründe des VIG sind vergleichbar, jedoch tendenziell enger als die des IFG.

Sonstige spezielle Informationszugangsregeln Schließlich gibt es noch eine Reihe weiterer spezieller Auskunftsrechte. Ein Anspruch auf Zugang zu Dokumenten der Europäischen Union ergibt sich beispielsweise aus Artikel 2 I der Verordnung (EG) Nr. 1049/2001. Darüber hinaus werden in Deutschland viele Register geführt, in welche meist voraussetzungslos jeder Einsicht erlangen kann, wie beispielsweise die Einsicht in das Vereinsregister gemäß § 79 BGB. Gemäß $\S 10$ I S. 1 BArchG steht jedermann auf Antrag das Recht zu, Archivgut des Bundes zu nutzen. Dafür ist weder ein berechtigtes Interesse noch die Verfolgung eines bestimmten Zwecks erforderlich (Debus 2017, IFG, § 1 Rn. 50).

\section{Sonderfall: Auskunftspflichten der Staatsanwaltschaft}

Von besonderer Praxisrelevanz ist die Frage, nach welchen gesetzlichen Regelungen Staatsanwaltschaften zur Pressearbeit berechtigt sind. Aufgrund des enormen öffentlichen Interesses an Strafermittlungsverfahren und der Tatsache, dass mittlerweile nahezu jede Staatsanwaltschaft über einen eigenen Pressesprecher verfügt, scheint diese Frage vielen geradezu ketzerisch. Tatsächlich erfordert jedoch gerade ein derart grundrechtssensibler Bereich wie das Strafermittlungsverfahren unmissverständliche Klarheit über Rechtsgrundlage, Anwendungsvoraussetzungen und Abwägungsmaßstäbe bei der behördlichen Medienarbeit. Denn während eines Strafermittlungsverfahrens ist der Beschuldigte ohnehin einer massiven staatlichen Übermacht ausgesetzt, die (unter Beachtung der jeweiligen Voraussetzungen) beispielsweise Telekommunikation abhören, Durchsuchungen durchführen oder Untersuchungshaft anordnen darf. In dieser besonderen Situation führen nahezu alle Äußerungen einer Staatsanwaltschaft in der Öffentlichkeit zu weiteren Eingriffen in die Grundrechte der Beschuldigten. Diese sind demgegenüber häufig schon deshalb nicht zu einer effektiveren medialen Verteidigung im Stande, weil sie nicht den Stand der Ermittlungen oder nicht ihrer strafprozessualen Einlassung vorgreifen möchten.

Trotz dieser großen und grundrechtssensiblen Bedeutung der Öffentlichkeitsarbeit von Ermittlungsbehörden enthält die Strafprozessordnung keine Bestimmung, unter welchen Voraussetzungen und auf welche Weise die Staatsanwaltschaft 
beispielsweise Presseanfragen zu Strafverfahren beantworten darf. Doch dass der Gesetzgeber die große praktische Relevanz derartiger Auskünfte schlicht übersehen hat, ist fernliegend: Schließlich enthalten die $\S \S 478 \mathrm{ff}$. StPO zahlreiche detailscharfe Regelungen zu Voraussetzungen und Reichweite von Auskunftsansprüchen anderer Behörden und Privater; eine versehentliche Regelungslücke scheint ausgeschlossen. Dementsprechend hat der Bundesgerichtshof (BeckRS 2006, 05100) in Bezug auf das IFG klargestellt, dass dessen Anwendung im Bereich der Strafrechtspflege aufgrund der spezielleren und abschließenden Regelungen der StPO ausgeschlossen ist (Debus 2017; § 1 Rn. 216.1). Vor diesem Hintergrund wäre es konsequent, auch in Bezug auf Presseanfragen zu Strafermittlungsverfahren einen Vorrang der strafprozessualen Spezialregelungen anzuerkennen und Auskunftsersuchen zu Strafermittlungsverfahren vom Anwendungsbereich der § 4 LPG auszunehmen.

Die Praxis der Verwaltungsgerichte (VGH Baden-Württemberg, Beschluss vom 16. Juni 2011 - 1 S 1137/11 -, BA S. 5 f.; VG Augsburg, Beschluss vom 29. Januar 2014 - Au 7 E 13.2018 -, juris Rn. 43) ist indes eine andere: Nach ganz allgemeiner Ansicht gewähren die allgemeinen presserechtlichen Auskunftsansprüche aus § 4 LPG auch gegenüber den Strafermittlungsbehörden grundsätzlich umfangreiche Auskunftsansprüche.

\section{Initiative Kommunikationspflichten der Verwaltung}

Seltener als die weit verbreiteten Pflichten zur Beantwortung von (Medien-) Anfragen sind die Fälle, in denen Behörden verpflichtet sind, von sich aus die Öffentlichkeit über bestimmte Sachverhalte zu informieren.

§ 31 II Produktsicherheitsgesetz (ProdSG) Gemäß § 31 I, II ProdSG sind die Marktüberwachungsbehörden und die Bundesanstalt für Arbeitsschutz und Arbeitsmedizin der Öffentlichkeit gegenüber verpflichtet, sie über Anordnungen, die die Rücknahme oder den Rückruf eines Produkts gebieten oder die eine Warnung der Öffentlichkeit verlangen (Tremml und Luber 2013, S. 262; Abs. 1), sowie über sonstige Erkenntnisse zu Produkten, die mit Risiken für die Sicherheit und Gesundheit von Personen verbunden sind, zu informieren.

§ 40 Abs. 1a Lebensmittel- und Futtermittelgesetzbuch (LFGB) Gemäß $\S 40$ Ia LFGB müssen die zuständigen Behörden die Öffentlichkeit informieren, wenn der hinreichend begründete Verdacht besteht, dass in Lebensmitteln zulässige 
Grenzwerte, Höchstgehalte oder Höchstmengen überschritten oder bestimmte verbraucherschützende Regelungen verletzt worden sind. Nachdem das Bundesverfassungsgericht mit Beschluss vom 21. März 2018 festgestellt hat, dass eine solche Information ohne zeitliche Beschränkung verfassungswidrig wäre, ist nunmehr eine zeitliche Begrenzung der Veröffentlichungsdauer der Warninformation geplant.

\section{Recht der Verwaltung auf Kommunikation}

Während also zahlreiche Gesetze bestimmte Kommunikationspflichten der Verwaltung regeln, bieten weder Verfassung noch das materielle Recht praktikable Antworten auf die Frage, unter welchen Voraussetzungen und gegebenenfalls in welchem Umfang eine Behörde berechtigt ist, sich - freiwillig - kommunikativ an die Öffentlichkeit zu wenden.

Keine Meinungsfreiheit zugunsten der Exekutive Zunächst ist festzustellen, dass die in Artikel 5 I GG verankerte Meinungs- und Pressefreiheiten weder unmittelbar noch mittelbar zu Gunsten von Verwaltungseinrichtungen Anwendung finden (Leibholz und Rinck 2018; Rn. 22, Art. 19 GG, Rn. 116, 119). Die Meinungsfreiheit ist primär ein subjektives Freiheitsrecht, das vor hoheitlichen Eingriffen schützt (Abwehrrecht) und Teilhabe am Meinungsbildungsprozess sichert (Teilhaberecht) (Isensee und Kirchhof 2009; § 162 Rn. 10). Weder aus der Abwehrfunktion noch aus dem grundrechtsimmanenten Teilhaberecht folgt jedoch ein eigenes Grundrecht des Staates auf Meinungsäußerungsfreiheit (Leibholz und Rinck 2018).

Recht auf Kommunikation qua Sachzusammenhang Gleichwohl soll es dem Staat grundsätzlich nach Auffassung des Bundesverfassungsgerichts auch ohne Berufung auf Artikel 5 GG und ohne spezialgesetzliche Rechtsgrundlagen möglich sein, sich mit eigenen Mitteilungen an die Öffentlichkeit zu wenden. Voraussetzung hierfür ist, dass die Äußerungen in einem unmittelbaren Sachzusammenhang mit einer Aufgabe stehen, die dem jeweiligen Hoheitsträger vom Gesetz zugewiesen ist, dass sie nicht als Ersatz für eine staatliche Maßnahme gelten, die als Grundrechtseingriff zu werten wäre (BVerfG NJW 2002, 2621, 2624), und dass sie die (weiter unten erläuterten) Mindestanforderungen an ihre inhaltliche Rechtmäßigkeit wahrt (BVerfG, Beschlüsse vom 24. Mai 2005 - 1 BvR 1072/01 -, BVerfGE 113, 63, und vom 26. Juni 2002 - 1 BvR 670/91 -, BVerfGE 105, 279). 
Ausnahme: Formale Rechtsgrundlage erforderlich Diese Äußerungsfreiheit der Behörden endet somit, wenn die mittelbaren Auswirkungen der öffentlichen Äußerung einem unmittelbaren Grundrechtseingriff gleichkommen (BVerfGE NJW 2002, 2621 ff.). Maßgeblich ist, ob die staatliche Äußerung ,,in der Zielsetzung und ihren Wirkungen Ersatz für eine staatliche Maßnahme ist, die als Grundrechtseingriff zu qualifizieren wäre." (BVerfGE NJW 2002, 2621, 2624) Dies ist beispielsweise der Fall, wenn eine Äußerung direkt auf die Marktbedingungen konkret individualisierter Unternehmen zielt, indem sie die Grundlagen der Entscheidungen am Markt zweckgerichtet beeinflusst und so die Markt- und Wettbewerbssituation zum wirtschaftlichen Nachteil der betroffenen Unternehmen verändert (BVerwG, Urteil vom 20. November 2014 - 3 C 27/13). Es liegt auf der Hand, dass der Staat nicht durch die Wahl eines in der Wirkung vergleichbaren, funktionellen Äquivalents (beispielsweise einer öffentlichen Produktwarnung anstelle eines Produktverbots) die besonderen Voraussetzungen der Rechtsordnung umgehen darf. In diesen Fällen setzt eine rechtmäßige Kommunikation der Behörde eine formale Rechtsgrundlage voraus.

\section{Inhaltliche Schranken der Kommunikation}

Die aus den vorangegangenen Überlegungen resultierende Feststellung, dass eine Behörde verpflichtet oder jedenfalls berechtigt sein kann, öffentlich zu kommunizieren, besagt jedoch noch nichts über die bei der Ausgestaltung dieser Kommunikation zu beachtenden rechtlichen Grenzen. Diese Grenzen setzen zum einen die von der Rechtsprechung aus dem Rechtsstaatsgebot abgeleiteten Mindestanforderungen rechtmäßiger Verwaltungskommunikation, zum anderen die Grundrechte der von den Äußerungen betroffenen Personen oder Unternehmen.

Funktionale Zuständigkeit Die erste Voraussetzung für die Zulässigkeit einer behördlichen Kommunikation ist, dass diese sich innerhalb des ihr vom Gesetz zugewiesenen Kompetenzrahmens bewegt. Dies gilt nicht nur dann, wenn sie spezialgesetzlich geregelt ist, sondern auch, wenn der Staat von der Möglichkeit Gebrauch macht, ohne spezielle Ermächtigung öffentlich Stellung zu beziehen (BVerfGE NJW 1977, 751, 753).

Insbesondere im letzteren Fall darf der Rahmen der behördlichen Kommunikationskompetenz jedoch nicht zu eng gefasst werden. So hat das Bundesverfassungsgericht bereits im Jahre 2002 in einer die Öffentlichkeitsarbeit der Bundesregierung betreffenden Entscheidung festgestellt, dass die Art und Weise der öffentlichen Kommunikation erheblichen Veränderungen unterliegt, denen auch der 
Staat gerecht werden muss (BVerfGE NJW 2002, 2621, 2623). Insbesondere wirke sich ,die gewachsene Rolle der Massenmedien, der Ausbau moderner Informationsund Kommunikationstechniken sowie die Entwicklung neuer Informationsdienste“ auf die Art und Weise der Kommunikation durch die Bundesregierung aus. So sei deren Öffentlichkeitsarbeit heutzutage nicht auf die bloße Darstellung aktueller Maßnahmen, Vorhaben und Aufgaben sowie das Werben um öffentliche Unterstützung hierfür beschränkt, sondern umfasse zum Beispiel die Aufgabe, die Öffentlichkeit über wichtige Vorgänge auch außerhalb oder weit im Vorfeld ihrer eigenen gestaltenden politischen Tätigkeit zu unterrichten:

„In einer auf ein hohes Maß an Selbstverantwortung der Bürger bei der Lösung gesellschaftlicher Probleme ausgerichteten politischen Ordnung ist von der Regierungsaufgabe auch die Verbreitung von Informationen erfasst, welche die Bürger zur eigenverantwortlichen Mitwirkung an der Problembewältigung befähigen. Dementsprechend erwarten die Bürger für ihre persönliche Meinungsbildung und Orientierung von der Regierung Informationen, wenn diese andernfalls nicht verfügbar wären.“(BVerfGE NJW 2002, 2621, 2623)

Übertragen auf die Praxis der Behörden bedeutet dies, dass diese nicht nur berechtigt sind, aktuelle Verwaltungsvorgänge zu kommunizieren, sondern sich darüber hinaus auch zu sonstigen, mit ihrem Aufgabengebiet zusammenhängenden, Sachverhalten öffentlich äußern dürfen.

\section{Neutralitätsgebot}

Darüber hinaus gehen Rechtsprechung und Literatur davon aus, dass die Verwaltung im Rahmen öffentlicher Äußerungen grundsätzlich verpflichtet ist, Neutralität zu waren. Verfassungsrechtlich wird diese Neutralitätspflicht zum einen auf das Gleichbehandlungsrecht (Artikel 3 Abs. 1 Grundgesetz) abgeleitet, zum anderen auf die abwehrrechtlichen Dimensionen der Freiheitsgrundrechte gestützt (Gusy 2015, S. 701). Tatsächlich liegt es nahe, dass es dem Staat grundsätzlich nicht erlaubt sein kann, im Rahmen wirtschaftlicher, ideeller, politischer oder sonstiger Wettbewerber einseitig Partei zu ergreifen.

Allerdings kann dies nur gelten, wenn die Verwaltung nicht selbst an den zu beurteilenden Sachverhalten beteiligt ist. Denn Neutralität kann nur von Außenstehenden erwartet werden, nicht hingegen von denjenigen, die innerhalb einer öffentlichen Debatte eine eigene Position vertreten. Umgekehrt bedeutet dies, dass Amtsträger, die (wie beispielsweise Landräte oder Bürgermeister) zugleich politische Repräsentanten der Bürger sind, durchaus Position ergreifen dürfen 
und Ereignisse, Zustände oder Veranstaltungen in ihrer Gemeinde billigen oder missbilligen und das gemeindliche Interesse oder Desinteresse an ihnen artikulieren dürfen (Gusy 2015, S. 702).

Keine Geltung kann das Neutralitätsgebot darüber hinaus beanspruchen, wenn die Verwaltung aufgrund einer gesetzlichen Regelung zur Kommunikation verpflichtet ist. Gesetzlich vorgesehene amtliche Informationen über Unternehmen, ihre Leistungen und deren (mögliche) Mängel sind auch zulässig, wenn dadurch auf die Wettbewerbssituation eines Unternehmens oder andere Grundrechtspositionen eingewirkt wird. Ebenso wie der Verfassungsschutzbericht ( $\$ 16$ II BVerfSchG) auf verfassungsfeindliche Bestrebungen auch unter Mitteilung von Parteinamen hinweisen darf, können Behörden verpflichtet sein, unter Nennung ihrer Handelsmarken vor Produkten zu warnen.

\section{Wahrheitsgebot}

Aus dem für sämtliche Staatsorgane verbindlichen Willkürverbot leitet das Bundesverfassungsgericht die Verpflichtung ab, sich wahrheitsgemäß zu äußern und mitgeteilte Tatsachen korrekt wiederzugeben (BVerfGE NJW 1981, 1359). Auch dieser auf den ersten Blick selbstverständliche Grundsatz wirft bei näherer Betrachtung mehrere Probleme auf.

Zum einen stellt sich die Frage, ob die infrage stehende Äußerung überhaupt als Tatsachenäußerung oder aber als Meinungsäußerung zu qualifizieren ist. Die aus dieser Unterscheidung resultierenden rechtlichen Konsequenzen sind immens: Denn während eine Tatsachenbehauptung stets objektiv wahr oder unwahr ist, drückt eine Meinungsäußerung nur die subjektive Bewertung eines Sachverhalts aus. Einer Meinungsäußerung kann ein Empfänger zwar zustimmen oder ihr widersprechen; da sie aber keine objektive Richtigkeit für sich beansprucht, kann sie nicht unwahr sein. Ausgangspunkt für die Qualifikation einer Äußerung als Tatsachenbehauptung ist daher die Frage, ob ihre (objektive) Richtigkeit mit den Mitteln des Beweises überprüfbar wäre (BGH NJW 2011, 2204 Rn. 10; EGMR NJW 2014, 3501). Hierbei kommt es nicht darauf an, ob die beweispflichtige Partei über hinreichende Beweismittel verfügt, sondern darauf, ob es - jedenfalls theoretisch irgendjemandem möglich wäre, die objektive Richtigkeit unter Beweis zu stellen. Hiervon ist jedenfalls dann auszugehen, wenn die Formulierung einer den im Zivilrecht geltenden Anforderungen genügenden Beweisfrage gelingt (BVerfG NJW-RR 2001, 411). Rechtliche Bewertungen sind hingegen im Regelfall keine dem Wahrheitsbeweis zugänglichen Behauptungen, sondern (insbesondere aus Sicht juristisch nicht vorgebildeter Adressaten) subjektive Bewertungen. 
Zum anderen stellt sich die Frage, ob und gegebenenfalls unter welchen Voraussetzungen eine Behörde auch dann zur Verbreitung von Informationen berechtigt sein kann, wenn deren Wahrheitsgehalt noch nicht abschließend feststeht. In diesen soll die staatliche Informationstätigkeit erlaubt sein, wenn die Information trotz ihrer nicht erwiesenen Wahrheit im öffentlichen Interesse liegt, wenn der Sachverhalt sorgfältig und unter Nutzung der verfügbaren Informationsquellen und gegebenenfalls unter Anhörung der Betroffenen aufgeklärt worden ist, und wenn die Adressaten auf die nicht erwiesene Richtigkeit der Information hingewiesen werden (VG Karlsruhe 6 K 7812/16, BeckRS 2017, 107428; BVerfGE 105, 252 [268 ff.] =NJW 2002，2621=NVwZ 2002，1495 L; BVerwGE 71, 183 [198 f.] = NJW 1985, 2774). Im Ergebnis sollen somit die im Zivilrecht entwickelten Grundsätze der Verdachtsberichterstattung auch auf behördliche Äußerungen Anwendung finden.

\section{Sachlichkeit und Verhältnismäßigkeit}

Dass Meinungsäußerungen, also Mitteilungen, die nicht als Tatsachenbehauptungen oder Verdachtsäußerungen einzuordnen sind, nie unwahr sein können, bedeutet nicht, dass sie schrankenlos erlaubt wären. Tatsächlich sind behördliche Meinungsäußerungen sogar an strengere Voraussetzungen gebunden als es vergleichbare Äußerungen von Privatpersonen wären. Der Grund hierfür liegt nicht nur in der fehlenden Grundrechtsfähigkeit der Behörden, sondern vor allem in dem für jede Art staatlichen Handelns geltenden Willkürverbot. Aus dem Willkürverbot leitet die Rechtsprechung das sogenannte Sachlichkeitsgebot ab. Demnach dürfen Werturteile nicht auf sachfremden Erwägungen beruhen, sondern müssen bei verständiger Beurteilung auf einem im Wesentlichen zutreffenden oder zumindest sachgerecht und vertretbar gewürdigten Tatsachenkern beruhen.

Ein wesentlicher Unterschied zu Äußerungen Privater ist jedoch, dass für behördliche Äußerungen das Verhältnismäßigkeitsgebot gilt (OVG Nordrhein-Westfalen, Beschluss vom 23.04.2012 - 13 B 127/12 -, NVwZ 2012, S. 767; BayVGH Beschluss vom 06.07.2012 - 4 B 12.952; OVG Bremen, 1 B 112/10, NJW 2010, 3738). Während im privaten Meinungskampf grundsätzlich auch unangemessene Meinungsäußerung erlaubt sind, muss die Meinungsäußerung einer Behörde geeignet, erforderlich und angemessen sein, um ein das von der Behörde verfolgte, legitime Ziel zu erreichen.

Kein Recht auf Gegenschlag Im öffentlichen Meinungskampf kommt es häufig zu einer wechselseitigen Verschärfung der Argumentation, der gegeneinander 
erhobenen Vorwürfe und ihrer Formulierungen. Zugunsten von privaten Rechtsträgern gewährt die Rechtsprechung daher unter dem als ,Recht auf Gegenschlag ‘ bezeichneten Rechtsinstitut einen teilweise erheblich größeren Spielraum rechtmäßiger Äußerungen.

Inzwischen hat das Bundesverfassungsgericht indessen klargestellt, dass das ,Recht auf Gegenschlag' nicht zugunsten von Behörden anwendbar ist. Anders als Private bleibt der Staat auch dann streng an die Einhaltung von Neutralität, Wahrhaftigkeit, Sachlichkeit und Verhältnismäßigkeit gebunden, wenn von anderer Seite überspitzte oder gar unzulässige Kritik geäußert wird. Ein „Recht staatlicher Organe auf unsachliche oder diffamierende Angriffe in gleicher Weise reagieren dürfen, besteht nicht.“ (BVerfG NJW 2018, 928, 931).

Rechte der Betroffenen Selbstverständlich müssen öffentliche Mitteilungen der Verwaltung auch den Rechten der inhaltlich betroffenen Personen und Unternehmen genügen. Dies sind in Bezug auf natürliche Personen insbesondere das aus Artikel 2 I in Verbindung mit 1 I GG abgeleitete allgemeine Persönlichkeitsrecht sowie die in Artikel 8 EMRK normierten Rechte auf Achtung des Privatund Familienlebens. Beim Schutz juristischer Personen kommt neben dem aus Artikel 2 I GG abgeleiteten Unternehmenspersönlichkeitsrecht vor allem den Grundrechten aus Artikel 12 I GG (Berufsfreiheit) und 14 GG (Eigentum) die größte praktische Relevanz zu.

\section{Literatur}

Barczak, T. (2015). Die parteipolitische Äußerungsbefugnis von Amtsträgern. Neue Zeitschrift für Verwaltungsrecht, 15, 1014-1020.

Debus, J. (2017). Rechtsschutz. In H. Gersdorf \& B. P. Paal (Hrsg.), BeckOK Informations- und Medienrecht (18. Aufl.). München: Beck. https://beck-online.beck.de/Dokument?vpath=bibdata\%5Ckomm\%5Cbeckokinfomedien_18\%5CIFG\%5Ccont $\% 5 \mathrm{CBE}$ CKOKINFOMEDIEN.IFG.P1.glF.htm. Zugegriffen: 1. Juni. 2019.

Gusy, C. (2015). Neutralität staatlicher Öffentlichkeitsarbeit - Voraussetzungen und Grenzen. Neue Zeitschrift für Verwaltungsrecht, 11, 700-704.

Isensee, J., \& Kirchhof, P. (Hrsg.). (2009). Handbuch des Staatsrechts der Bundesrepublik Deutschland: Bd. VII. Freiheitsrechte (3. Aufl.). Heidelberg: C. F. Müller.

Karg, M. (2018). Bedeutsame Schutzgüter der öffentlichen Sicherheit. In H. Gersdorf \& B. P. Paal (Hrsg.), BeckOK Informations- und Medienrecht (24. Aufl.). München: Beck. https://beck-online.beck.de/Dokument?vpath=bibdata\%2Fkomm\%2Fbeckokinfomedien_24\%2Fuig\%2Fcont\%2Fbeckokinfomedien.uig.p8.glc.glii.gl3.htm\&pos=8. Zugegriffen: 1. Juni. 2019. 
Leibholz, G., \& Rinck, H.-J. (Hrsg.). (2018). Grundgesetz - Rechtsprechung des BVerfG. Kommentar, 77. Aktualisierung 2018. Köln: Schmidt.

Löffler, M., Wenzel, K. E., Sedelmeier, K., \& Burkhardt, E. H. (Hrsg.). (2015). Presserecht - Kommentar zu den deutschen Landespressegesetzen (6. Aufl.). München: Beck.

Paschke, M., Berlit, W., \& Meyer, C. (Hrsg.). (2016). Hamburger Kommentar gesamtes Medienrecht (3. Aufl.). Baden-Baden: Nomos.

Schnabel, C. (2016). Die Zukunft des presserechtlichen Auskunftsanspruchs gegen Bundesbehörden. Neue Juristische Wochenschrift, 24, 1692-1696.

Tremml, B., \& Luber, M. (2013). Amtshaftungsansprüche wegen rechtswidriger Produktwarnungen. Neue Juristische Wochenschrift, 5, 262-267.

Open Access Dieses Kapitel wird unter der Creative Commons Namensnennung 4.0 International Lizenz (http://creativecommons.org/licenses/by/4.0/deed.de) veröffentlicht, welche die Nutzung, Vervielfältigung, Bearbeitung, Verbreitung und Wiedergabe in jeglichem Medium und Format erlaubt, sofern Sie den/die ursprünglichen Autor(en) und die Quelle ordnungsgemäß nennen, einen Link zur Creative Commons Lizenz beifügen und angeben, ob Änderungen vorgenommen wurden.

Die in diesem Kapitel enthaltenen Bilder und sonstiges Drittmaterial unterliegen ebenfalls der genannten Creative Commons Lizenz, sofern sich aus der Abbildungslegende nichts anderes ergibt. Sofern das betreffende Material nicht unter der genannten Creative Commons Lizenz steht und die betreffende Handlung nicht nach gesetzlichen Vorschriften erlaubt ist, ist für die oben aufgeführten Weiterverwendungen des Materials die Einwilligung des jeweiligen Rechteinhabers einzuholen.

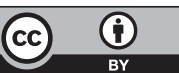

